\title{
Uniqueness of approximate solutions to the Gelfand Levitan equation
}

\author{
Eric A. Kincanon * \\ Gonzaga University, Spokane, WA USA \\ *Corresponding author E-mail: kincanon@gonzaga.edu
}

\begin{abstract}
This brief paper considers a potential issue of using iterative solutions for the Gelfand-Levitan equation. Iterative solutions require approximation methods and this could lead to a loss of uniqueness of solutions. The calculations in this paper demonstrate that this is not the case and that uniqueness is preserved.
\end{abstract}

Keywords: Gelfand-Levitan Equation; Inverse Scattering; Ambiguities; Approximation Methods.

\section{Introduction}

The Gelfand-Levitan equation [1-3]:

$K(r, s)+G(r, s)+\int_{-\infty}^{r} K(r, t) G(t, s) d t=0$

is used to model inverse scattering problems on the line. The potential $\mathrm{V}(\mathrm{r})$ and the reflection coefficient $\mathrm{R}(\mathrm{k})$ are given by:

$$
\begin{aligned}
& V(r)=-2 \frac{d K(r, r)}{d r} \\
& G(r, s)=\frac{1}{2 \pi} \int_{-\infty}^{+\infty} R(k) e^{-i k(r+s)} d k
\end{aligned}
$$

The typical problem is to determine the potential given the reflection coefficient. In practice this can be quite complicated since the GelfandLevitan equation (GL) is a non-linear integral equation. One common method [4] for solving this problem is to rewrite GL as:

$K(r, s)=-G(r, s)-\int_{-\infty}^{r} K(r, t) G(t, s) d t$

and then iterate to give:

$K(r, s)=-G(r, s)+\int_{-\infty}^{r} G(r, t) G(t, s) d t-\int_{-\infty}^{r} G(r, t) \int_{-\infty}^{r} G(t, u) G(u, s) d u+$ continued terms of iteration

This iteration is continued out until a pattern is found to identify a function the series converges to or the terms become small enough to be ignored. So, this is usually an approximating method.

Here, this method is considered with respect to potential ambiguities to see if the approximation could lead to the case of two $\mathrm{G}(\mathrm{r}, \mathrm{s})$ corresponding to the same potential. In other words, could the approximation method lead to non-unique solutions?

\section{Calculations}

Consider GL for two different $G(r, s)$ : $G_{1}(r, s)$ and $G_{1}(r, s)+G_{2}(r, s)$. Equation (4) for each of these would be:

$$
\begin{aligned}
& K(r, s)=-G_{1}(r, s)-\int_{-\infty}^{r} K(r, t) G_{1}(t, s) d t \\
& K^{*}(r, s)=-G_{1}(r, s)-G_{2}(r, s)-\int_{-\infty}^{r} K^{*}(r, t)\left[G_{1}(t, s)+G_{2}(t, s)\right] d t
\end{aligned}
$$


Subtracting equation (7) from (6) and grouping like $G(r, s)$ terms yields:

$K(r, s)-K^{*}(r, s)=G_{2}(r, s)-\int_{-\infty}^{r}\left[K(r, t)-K^{*}(r, t)\right] G_{1}(t, s) d t+\int_{-\infty}^{r}\left[K^{*}(r, t)\right] G_{2}(t, s) d t$

Defining $\mathrm{DK}$ and $\mathrm{K}-\mathrm{K}^{*}$ gives:

$\Delta K(r, s)=G_{2}(r, s)-\int_{-\infty}^{r}[\Delta K(r, t)] G_{1}(t, s) d t+\int_{-\infty}^{r}\left[K^{*}(r, t)\right] G_{2}(t, s) d t$

Iterating this equation yields:

$\Delta K(r, s)=G_{2}(r, s)-\int_{-\infty}^{r} G_{2}(r, t) G_{1}(t, s) d t+$ other iterative terms

Key to this discussion is that close examination of the "other iterative terms" show that they can be grouped such that they are multiples of the first two terms on the right-hand side of equation (10). In other words, all of the right-hand side terms of equation (10) look like:

Function $*\left\{G_{2}(r, s)-\int_{-\infty}^{r} G_{2}(r, t) G_{1}(t, s) d t\right\}$

So now DK can be studied by looking at the characteristics of the term in the braces in (11). DK is the difference between the two K's that were associated with the two different $G(r, s)$ terms. So if a $G_{1}(r, s)$ and $G_{2}(r, s)$ can be identified such that DK is small then there would be an ambiguity associated with the iterative method.

To explore this, consider the method of solving GL using the representation:

$\mathrm{G}_{1}(\mathrm{r}, \mathrm{s})=\sum_{\mathrm{n}=1}^{\infty} \beta_{\mathrm{n}} \mathrm{e}^{\mathrm{n}(\mathrm{r}+\mathrm{s})}$

And

$\mathrm{G}_{2}(\mathrm{r}, \mathrm{s})=\sum_{\mathrm{m}=1}^{\infty} \gamma_{\mathrm{m}} \mathrm{e}^{\mathrm{m}(\mathrm{r}+\mathrm{s})}$

These assumed forms of G(r,s) have been shown to be useful in an general method [5-6] of solving GL and so it is reasonable to assume these mathematical representations.

Now looking at the second term in the braces of equation (11) and applying equations (12) and (13) gives:

$\int_{-\infty}^{r} G_{2}(r, t) G_{1}(t, s) d t=\int_{-\infty}^{r} \sum_{n=1}^{\infty} \beta_{n} e^{n(r+t)} \sum_{m=1}^{\infty} \gamma_{m} e^{m(t+s)} d t$

$=\sum_{n=1}^{\infty} \sum_{m=1}^{\infty} \beta_{n} \gamma_{m} e^{n r} e^{m s} \int_{-\infty}^{r} e^{(n+m) t} d t$

$=\sum_{\mathrm{n}=1}^{\infty} \sum_{\mathrm{m}=1}^{\infty} \beta_{\mathrm{n}} \gamma_{\mathrm{m}} \frac{1}{\mathrm{n}+\mathrm{m}} \mathrm{e}^{2 \mathrm{nr}} \mathrm{e}^{\mathrm{m}(\mathrm{r}+\mathrm{s})}$

Using this expression and equation (13), the term in the braces of equation (11) is:

\{\}$=\sum_{\mathrm{n}=1}^{\infty} \beta_{\mathrm{n}} \mathrm{e}^{\mathrm{nr}}\left[\mathrm{e}^{\mathrm{ns}}-\mathrm{e}^{\mathrm{nr}} \sum_{\mathrm{m}=1}^{\infty} \gamma_{\mathrm{m}} \frac{1}{\mathrm{n}+\mathrm{m}} \mathrm{e}^{\mathrm{m}(\mathrm{r}+\mathrm{s})}\right]$

The issue here is whether the left-hand side of this equation can be small because that would point to an ambiguity in the solution method. For this to be the case it must be that the term in brackets is small. So, consider when

$\mathrm{e}^{\mathrm{ns}}-\mathrm{e}^{\mathrm{nr}} \sum_{\mathrm{m}=1}^{\infty} \gamma_{\mathrm{m}} \frac{1}{\mathrm{n}+\mathrm{m}} \mathrm{e}^{\mathrm{m}(\mathrm{r}+\mathrm{s})}$

is small.

Note that the first term in the above is s-dependent only. In order for this to be small it must be that the second term also is only s-dependent. This leads to the requirement that:

$\frac{\mathrm{d}}{\mathrm{dr}}\left[\mathrm{e}^{\mathrm{nr}} \sum_{\mathrm{m}=1}^{\infty} \gamma_{\mathrm{m}} \frac{1}{\mathrm{n}+\mathrm{m}} \mathrm{e}^{\mathrm{m}(\mathrm{r}+\mathrm{s})}\right]=0$

Taking this derivative and rearranging terms yields:

$\mathrm{e}^{\mathrm{nr}} \sum_{\mathrm{m}=1}^{\infty} \gamma_{\mathrm{m}} \mathrm{e}^{\mathrm{m}(\mathrm{r}+\mathrm{s})}=0$

But now there is a serious issue. The summation term is $\mathrm{G}_{2}(\mathrm{r}, \mathrm{s})$, giving:

$\mathrm{e}^{\mathrm{nr}} \mathrm{G}_{2}(\mathrm{r}, \mathrm{s})=0$

Which can only be true if $\mathrm{G}_{2}(\mathrm{r}, \mathrm{s})=0$. Leaving us with no difference between the two assumed forms of $\mathrm{G}(\mathrm{r}, \mathrm{s})$ in equations (6) and (7). 


\section{Conclusion}

The uniqueness of analytic solutions to the Gelfand-Levitan equation have been clearly demonstrated [3, 7]. There could still be a concern however since being a non-linear equation, the Gelfand-Levitan equation can be very difficult to solve analytically. In using approximation methods to solve the Gelfand-Levitan equation there could be the concern that the uniqueness of the equation's solution is not preserved. This brief paper has shown that this is not the case for iterative solutions and that no ambiguities exist.

\section{References}

[1] I.M. Gelfand, B.M. Levitan, On the determination of a differential equation by its spectral function, Dokl. Akad. Nauk. USSR 77 (1951) 557-560.

[2] I.M. Gelfand, B.M. Levitan, On the determination of a differential equation by its spectral measure function, Izv. Akad. Nauk. SSR 15 (1951) 309360

[3] K. Chadan, P.C. Sabatier, Inverse Problems in Quantum Scattering Theory, Springer-Verlag, New York, 1977. https://doi.org/10.1007/978-3-662$12125-2$

[4] R. Jost, W. Kohn, On the relation between phase shift energy levels and the potential, Danske Vid. Selsk. Math. Fys. 27 (1953) 3 -19.

[5] E. Kincanon, An Orthogonal Set Composed from the Functions enx, Applied Mathematics and Computation, 41, (1991) 69-75. https://doi.org/10.1016/0096-3003(91)90107-X.

[6] E. Kincanon, Approximate solution it the Gelfand-Levitan equation, Applied Mathematics and Computation, 53 (1993) 121-128. https://doi.org/10.1016/0096-3003(93)90097-X.

[7] I. Kay, H.E. Moses, A Simple Verification of the Gelfand-Levitan Equation for the Three-Dimensional Scattering Problem, Communications on Pure and Applied Mathematics, 24 (1961) 435-445. https://doi.org/10.1002/cpa.3160140319. 